\title{
The blue straggler population of the old open cluster Berkeley 17
}

\author{
Souradeep Bhattacharya (মৌরদীপ ভট্টাচার্য্য) ${ }^{1}$, Kaushar Vaidya ${ }^{2}$, W. P. Chen ${ }^{3}$, and Giacomo Beccari ${ }^{1}$ \\ 1 European Southern Observatory, Karl-Schwarzschild-Str. 2, 85748 Garching, Germany \\ e-mail: sbhattac@eso.org \\ 2 Department of Physics, Birla Institute of Technology and Science, Pilani 333031, Rajasthan, India \\ ${ }^{3}$ Graduate Institute of Astronomy, National Central University, 300 Jhongda Road, Jhongli 32001, Taiwan
}

Received 17 October 2018 / Accepted 15 February 2019

\begin{abstract}
Context. Blue straggler stars (BSSs) are observed in Galactic globular clusters and old open clusters. The radial distribution of BSSs has been used to diagnose the dynamical evolution of globular clusters. For the first time, with a reliable sample of BSSs identified with Gaia DR2, we conduct such an analysis for an open cluster.

Aims. We aim to identify members, including BSSs, of the oldest known Galactic open cluster Berkeley 17 with the Gaia DR2 proper motions and parallaxes. We study the radial distribution of the BSS population to understand the dynamical evolution of the cluster. Methods. We selected cluster members to populate the colour magnitude diagram in the Gaia filters. Cluster parameters are derived using the brightest members. The BSSs and giant branch stars are identified, and their radial distributions are compared. The segregation of BSSs is also evaluated with respect to the giant branch stars using the minimum spanning tree (MST) analysis.

Results. We determine Berkeley 17 to be at $3138.6_{-352.9}^{+285.5}$ pc. We find 23 BSS cluster members, only two of which were previously identified. We find a bimodal radial distribution of BSSs supported by findings from the MST method.

Conclusions. The bimodal radial distribution of BSSs in Berkeley 17 indicates that they have just started to sink towards the cluster centre, placing Berkeley 17 with globular clusters of intermediate dynamical age. This is the first such determination for an open cluster.
\end{abstract}

Key words. blue stragglers - open clusters and associations: individual: Berkeley 17 - methods: data analysis

\section{Introduction}

In the colour magnitude diagrams (CMDs) of globular clusters and old open clusters, blue straggler stars (BSSs) are observed to be brighter and bluer than the main sequence turn-off lying along an extrapolation of the main sequence (Sandage 1953). Mass transfer in a binary system (McCrea 1964) and direct stellar collisions (Hills \& Day 1976) have been considered to be the possible physical mechanism for BSS formation. BSS populations and their formation scenarios in globular clusters have been the subject of many studies to understand their nature and evolution in the context of known stellar evolution processes (e.g. Davies et al. 2004; Ferraro et al. 2009; Sills et al. 2013).

Irrespective of their formation mechanism, BSSs are more massive than giant branch stars and their radial distribution has been used as a "dynamical clock" for globular clusters to measure the level of dynamical evolution of the system. Ferraro et al. (2012) showed that based on the BSS radial distribution with respect to that of the light (or reference stars), globular clusters can be classified into three families: Family I globular clusters have a flat distribution and are "dynamically young"; Family II globular clusters have a bimodal distribution, with a peak at the cluster centre, a minimum at intermediate radii, a rise in the external regions (e.g. Dalessandro et al. 2009; Beccari et al. 2012), and they are evolving under efficient dynamical friction; Family III globular clusters have a central peak followed by a monotonically decreasing trend and are "dynamically old".

In the case of old open clusters, while a large number of BSSs have been reported (Ahumada \& Lapasset 2007), their numbers remain sparse with uncertain membership except for the very close open clusters (e.g. NGC 188; Mathieu \& Geller 2009, M 67; Bertelli Motta et al. 2018). It has thus not been possible to study BSSs in open clusters as thoroughly as in globular clusters, since their radial distribution remains elusive with uncertain membership. Possible formation mechanisms have been examined only for the very close open clusters. However, the sparse nature of open clusters makes them ideal laboratories in which to study the nature and formation of BSSs with spectroscopy.

Berkeley $17(\mathrm{RA}=$ 05:20:37, $\mathrm{Dec}=+30: 35: 12$, J2000, hereafter Be17) is located near the Galactic anti-centre at a distance of $\sim 2.7 \mathrm{kpc}$ (Phelps 1997). With a metallicity of $[\mathrm{Fe} / \mathrm{H}] \approx-0.33$ (Friel et al. 2002) and an age of $\sim 10$ Gyr (Kaluzny 1994; Phelps 1997; Salaris et al. 2004; Krusberg \& Chaboyer 2006), it is the oldest known open cluster. While Bragaglia et al. (2006) found a slightly lower age of 8.5-9 Gyr, these authors did not rule out an older age up to $\sim 12 \mathrm{Gyr}$. It is mass segregated and has a distinct tidal tail structure (Chen et al. 2004; Bhattacharya et al. 2017) possibly resulting from influence by the Perseus arm of the Galaxy. It was also known to be rich in BSSs with 31 candidates identified by Ahumada \& Lapasset (2007), though Bhattacharya et al. (2017) showed that around half of those might be field stars. Being close to the Galactic plane, Be17 has a significant field contamination, which in the CMD occupies the same region as the BSSs. Thus, membership determination is essential to study the BSSs in Be17.

With the second Data Release of Gaia (Gaia Collaboration 2018, hereafter GDR2), accurate proper motions and parallaxes have become available to allow us to identify the bright members 
of Be17, including the BSSs. We can thus for the first time use the BSS population to probe the dynamical state of an old open cluster, following the method suggested by Ferraro et al. (2012). It is important to identify whether BSSs are efficient test particles to infer the dynamical state of a stellar system like an open cluster, which offers a completely different environment (in terms of total mass, formation history, and stellar density) as compared to globular clusters. We describe the data used in Sect. 2 to determine the cluster members in Sect. 3. We derive the properties of the cluster in Sect. 4 and characterize the BSS population and its radial distribution in Sect. 5. Finally, we end with a discussion in Sect. 6.

\section{Data description}

GDR2 provides the position, trigonometric parallax, and proper motion as well as photometry in three broad-band filters (G, GBP, and GRP) for more than a billion stars. It also provides spectroscopic radial velocities for the brightest stars. The astrometric solution is described in Lindegren et al. (2018), the photometric content and validation is described in Evans et al. (2018), while the spectroscopy is described in Katz et al. (2019). From the known centre of Be17, its core region and tidal tails are within a radius of $\sim 11^{\prime}$ (Bhattacharya et al. 2017). So we used GDR2 data of 8357 stars within a slightly larger $15^{\prime}$ radius from the known centre of Be17 for our analysis.

\section{Membership determination}

We use a procedure similar to Yen et al. (2018) to identify Be17 cluster members based on the GDR2 proper motions and parallaxes. The GDR2 counterparts of previously known members are first identified. To identify possible members of Be17 with GDR2, we selected stars within three times the standard deviation of the mean proper motion of the known members. We only selected those sources whose proper motion errors are within $0.5 \mathrm{mas} \mathrm{yr}^{-1}$, where mas stands for milli-arcsecond, to ensure accuracy. Of the sources thus selected, we further considered as members only those whose trigonometric parallax values are within 1.5 times the standard deviation of the known members.

Scott et al. (1995) identified 13 giant stars as members of Be17 based on their location on the CMD and radial velocities. Of these, only 12 were confirmed as members by Friel et al. (2002) based on their metallicity, $[\mathrm{Fe} / \mathrm{H}]$, which was determined for each star from spectroscopic indices that primarily measured $\mathrm{Fe} I$ and $\mathrm{Fe}$-peak blends. We identify the GDR2 counterparts to these stars as the nearest neighbours within $0.2^{\prime \prime}$. We find the mean proper motion of these 12 giants to be $\mu_{\alpha} \cos \delta=2.71 \pm$

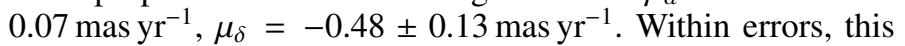
is close to the previously adopted uncertain value of $\mu_{\alpha} \cos \delta=$ $3.60 \pm 3.34$ mas yr$^{-1}, \mu_{\delta}=-3.62 \pm 2.27$ mas yr$^{-1}$ obtained by Dias et al. (2014) from proper-motion-selected members from UCAC4 (the fourth U.S. Naval Observatory CCD Astrograph Catalog). The increased accuracy is a testament of the quality of the GDR2 data. We also find the mean trigonometric parallax of the 12 giants as $\bar{\omega}=0.3 \pm 0.03$ mas.

Proper-motion-selected members of Be17 are those within three times the standard deviation of the mean proper motion of the known giants whose errors on the $\mu_{\alpha} \cos \delta$ and $\mu_{\delta}$ are within $0.5 \mathrm{mas} \mathrm{yr}^{-1}$. Figure 1 shows the 523 proper-motionselected stars in blue while all the stars within $15^{\prime}$ from the centre of Be17 are shown in grey. The BSS candidates identified by Ahumada \& Lapasset (2007) are shown in orange.
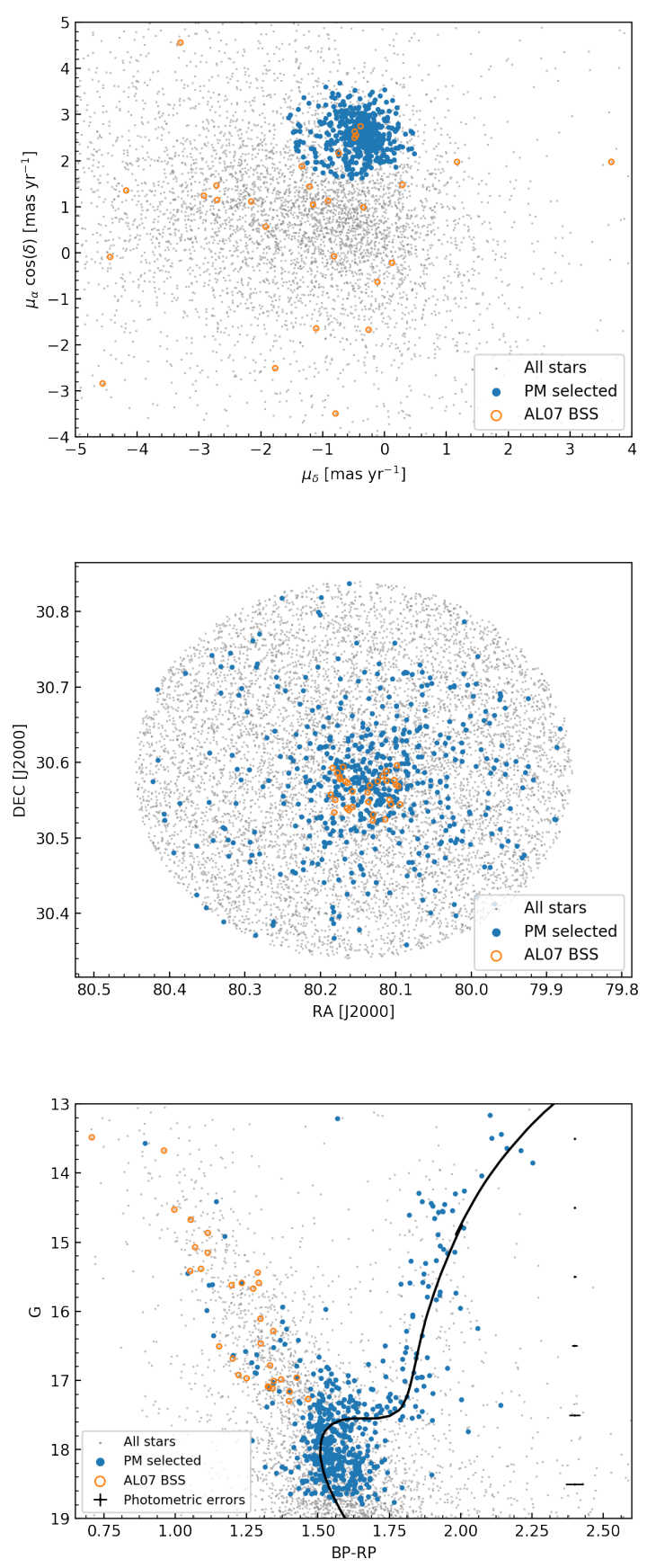

Fig. 1. All the sources within $15^{\prime}$ from the known centre of Be17 are shown in grey, the proper-motion-selected members are shown in blue, and the BSS candidates from Ahumada \& Lapasset (2007) are shown in orange. Top: proper motion of the stars. Middle: spatial distribution of the stars. Bottom: CMD for the stars where the isochrone, shown with a black solid line, corresponds to the adopted age and metallicity of Be17. The photometric errors are shown on the right in black. The errors in magnitude are negligible.

Only five of them are classified as members in the proper motion selection, as evident in the top panel of Fig. 1. The middle panel of Fig. 1 shows the spatial distribution of the stars where the proper-motion-selected members appear centrally concentrated with an apparent cluster halo. The stars at the outskirts of the spatial distribution may be non-members. From the CMD shown in the lower panel of Fig. 1, it is evident that the proper-motion-selected members follow the isochrone (described later in Sect. 4) suitable for Be17. The overlap of field 

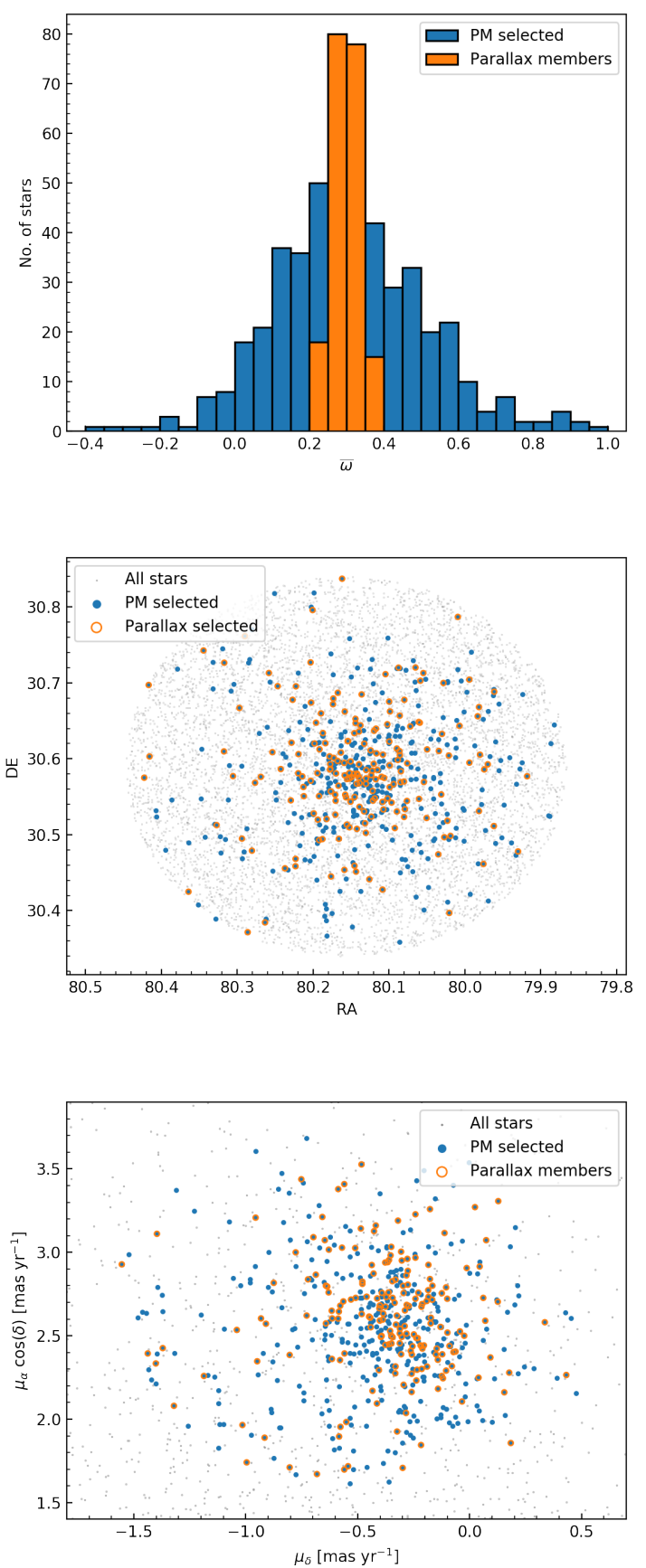

Fig. 2. Top: proper-motion-selected members are shown with a blue histogram while the parallax-selected members are shown with an orange histogram. Middle: spatial distribution of the stars with all the sources within $15^{\prime}$ from the known centre of Be17 shown in grey, the proper-motion-selected members shown in blue, and the parallaxselected members shown in orange. Bottom: proper motion of the same stars.

stars in the region of the CMD occupied by BSSs is evident and this resulted in the misidentification of field stars as BSSs by Ahumada \& Lapasset (2007). To further remove field contamination, the membership determination may be polished by parallax selection of cluster members.

Of the proper-motion-selected members, only those whose trigonometric parallax values are within 1.5 times the standard deviation of the known giants are considered as cluster members. With a more relaxed parallax selection, more faint sources with higher parallax uncertainties would have been selected.
Therefore, in order to limit contamination from field stars, the stringent selection criteria in parallax was applied. We did not apply an error cut to the parallax selection to avoid being biased towards more nearby sources, which have less uncertainties in their parallax determination. The parallax selection is shown in the top panel of Fig. 2. In total we identify 191 sources as members of Be17. The spatial distribution of stars shown in the middle panel of Fig. 2 still shows that some of the identified cluster members are on the outskirts of the search area. The lower panel of Fig. 2 shows that a majority of the parallax-selected members showed a concentration in the proper motion space slightly offset from the mean proper motion of the known giants, indicating that the mean proper motion of $\mathrm{Be} 17$ is slightly different from that of the known giants members. A few stars appear further away from this concentration but it is a significant improvement from just the proper motion selection. These may indeed be field contaminants and the membership selection may be further refined with the next Gaia data release. The membership determination is accurate enough to study the bright sources in Be17, including the BSSs.

\section{Cluster properties}

Of the identified members, six sources have GDR2 radial velocity information from low resolution spectrophotometry, giving a mean radial velocity, $V_{\text {rad }}=-72.86 \pm 0.98 \mathrm{~km} \mathrm{~s}^{-1}$. This is close to $V_{\mathrm{rad}}=-84 \mathrm{~km} \mathrm{~s}^{-1}$ derived by Scott et al. (1995) for their 12 giants within their estimated standard deviation, $\sigma_{\mathrm{v}}=11 \mathrm{~km} \mathrm{~s}^{-1}$. Three of these bright GDR2 sources are the counterparts of the known giants with IDs 4607, 0079 and 0099 in the catalogue of Scott et al. (1995). Their individual radial velocities match within errors as Scott et al. (1995) note that each of their individual radial velocity measurements may have an uncertainty of $\sim 10 \mathrm{~km} \mathrm{~s}^{-1}$. For the six sources observed with spectrophotometry, GDR2 also provides extinction in the $G$-filter, $A_{G}$, and reddening, $E(B P-R P)$, inferred using the Apsis-Priam system (Bailer-Jones et al. 2013). We find a mean $A_{G}=1.514$ and mean reddening $E(B P-R P)=0.7358$ for these six sources.

Since reliable distances to the GDR2 sources cannot be obtained by simply inverting the parallax, Bailer-Jones et al. (2018) use an inference procedure to obtain distances for each of the GDR2 sources accounting for the non-linearity of the transformation and the asymmetry of the resulting probability distribution. The mean distance of $\mathrm{Be} 17$ is the mean of the individual source distances obtained by Bailer-Jones et al. (2018) for the brightest cluster members that have $G<15 \mathrm{mag}$. This gives $3138.6_{-352.9}^{+285.5} \mathrm{pc}$, in good agreement with that found by Cantat-Gaudin et al. (2018) also using GDR2 but selecting cluster members with a different method. We use only the brightest members because the uncertainties on parallax are much larger for the fainter sources. The derived distance to Be17 is slightly farther than the previously known value of $2.7 \mathrm{kpc}$ (Phelps 1997).

For all the identified members, we obtain the CMD in the GDR2 colours shown in Fig 3. The PARSEC stellar evolution isochrone $^{1}$ (Bressan et al. 2012; Chen et al. 2014; Marigo et al. 2017) for the Gaia filters has been plotted corresponding to the $10 \mathrm{Gyr}$ age of Be17, its metallicity of $0.007([\mathrm{Fe} / \mathrm{H}]=-0.33)$, the obtained distance, and the obtained $A_{G}$ and $E(B P-R P)$. The location of the cluster members, both the giant branch and the BSSs, on the CMD is well reproduced by the isochrone.

1 http://stev.oapd.inaf.it/cgi-bin/cmd 


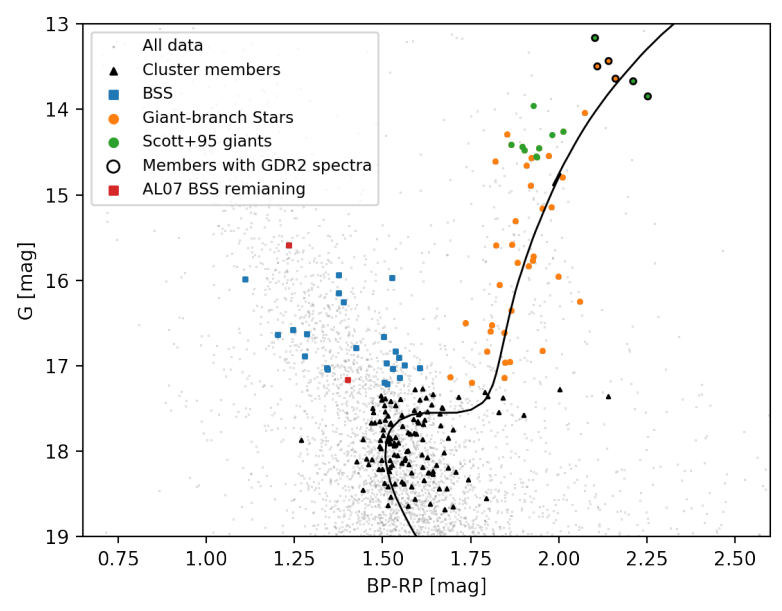

Fig. 3. Colour magnitude diagram showing cluster members in black triangles while all sources in the search area are shown in grey. The cluster members along the giant branch are shown in orange while those shown as blue squares are the BSSs. The two dark red squares are BSSs identified by Ahumada \& Lapasset (2007) and re-identified in this work. The 12 giants from Scott et al. (1995) are shown in green and are re-identified as cluster members in this work. The six stars with spectrophotometric data from GDR2 are circled in black. The PARSEC isochrone, shown with a black solid line, corresponds to the adopted age and metallicity of Be17.

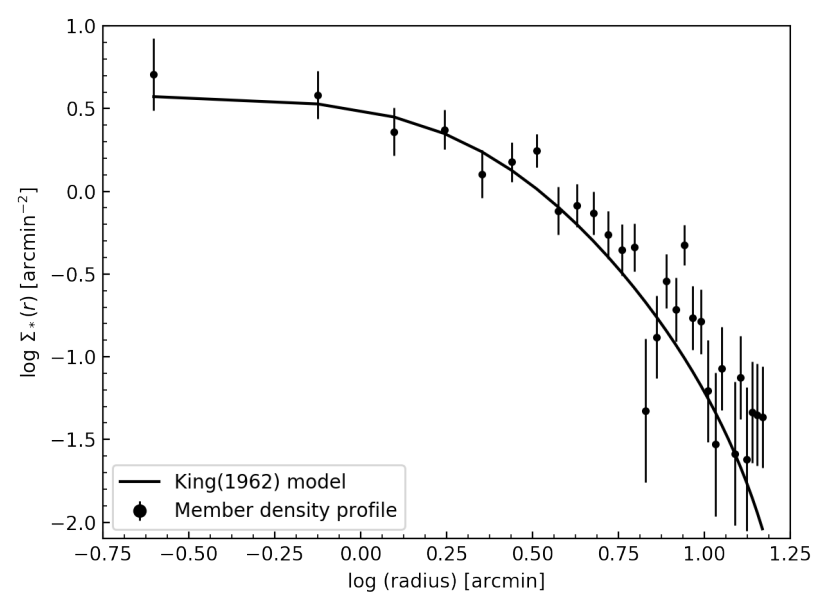

Fig. 4. Radial density profile of member stars in equi-radial bins fitted with an isotropic single-mass King model given by King (1962).

From the isochrone, we find that the stars at the main sequence turn-off have masses $\sim 0.9 M_{\odot}$ while the stars along the giant branch have masses $\sim 0.9-1 M_{\odot}$. While the evolutionary status of BSSs is uncertain, their single-star masses are inferred as the main sequence turn-off mass corresponding to the PARSEC stellar evolution isochrones with the distance and metallicity appropriate for $\mathrm{Be} 17$ but of lower ages. The inferred masses are in the range $\sim 1-1.6 M_{\odot}$, more than those of the giant branch stars, corresponding to the main sequence turn-off mass of the isochrone with turn-off passing through the faintest and brightest identified BSS, respectively.

Since the radial distribution of BSSs (discussed in Sect. 5) is highly dependent on the position of the cluster centre, we estimated the centre of Be17 by finding the centroid of all members in different magnitude bins of $0.5 \mathrm{mag}$ from $15.5 \mathrm{mag}$ to $18 \mathrm{mag}$, such that each bin has a statistically significant number of cluster members. We obtained the centre of Be17 from the mean of the centroid positions of each bin as $\mathrm{RA}=05: 20: 33.67$,
Dec $=+30: 35: 08.71($ J2000) accurate within 15". We also constructed the radial density profile of the cluster by dividing all the observed cluster members into 30 bins at equal radial intervals and computing the number density of member stars in each bin. The resulting profile (as shown in Fig. 4) is nicely fitted with an isotropic single-mass King model given by King (1962). The fitting provides the normalization factor $(k)$, core $\left(r_{\mathrm{c}}\right)$, and tidal $\left(r_{\mathrm{t}}\right)$ radii as $4 !^{\prime 2} 77 \pm 1 !^{2} 21,2.28 \pm 0.75$, and $20.76 \pm 9.49$ respectively. While the uncertainty is large, within errors the parameters determined are close to those found by Kharchenko et al. (2013), $k=6 .^{\prime 2} 28, r_{\mathrm{c}}=1.2$, and $r_{\mathrm{t}}=6.94$, estimated without determining membership for Be17. They report errors as negligible but most stars in their observed field were non-members.

\section{Blue straggler population}

Interestingly, of the 31 sources identified as BSSs in Be17 by Ahumada \& Lapasset (2007, tabulated in Chen et al. 2017), only two are identified by us as members. The majority of them were already classified as non-members in the proper motion selection (Fig. 1) and only two survived the parallax selection (Fig. 3). This is not surprising because Ahumada \& Lapasset (2007) did not employ any membership criteria in selecting the BSSs and since the background stars of Be17 occupy the same area in the CMD as the BSSs do (Bhattacharya et al. 2017), reliable identification of BSSs is not possible without any membership constraints. This effect of field-star contamination on the BSSs identified by Ahumada \& Lapasset (2007) had also been seen by Carraro et al. (2008) for the open clusters NGC 7789, Berkeley 66 , and Berkeley 70 .

\subsection{Radial distribution}

Being more massive than most cluster members, the BSSs should appear more centrally concentrated in a dynamically evolved star cluster as a result of mass segregation. Mass segregation is already observed in the core of $\mathrm{Be} 17$ (Bhattacharya et al. 2017) showing that it is certainly undergoing dynamical relaxation. The accurate astrometric capabilities of GDR2 enable us to identify reliably the stellar population belonging to Be17 from the tip of the red giant branch (RGB) down to the main sequence turn-off. We thus selected the BSSs (shown in squares in Fig. 3) of the cluster, and compared their radial distribution with those along the RGB (shown in orange and green in Fig. 3), chosen as the reference population, to understand the dynamical evolution of Be17. We identified 23 BSSs in Be17. In addition we selected 45 RGB stars as the reference population with the same magnitude depth as the BSSs, so as to ensure both samples are equally affected by incompleteness.

We first compared the cumulative radial distribution of the BSS population to that of the reference stars to conduct a Kolmogorov-Smirnov test, which yields a probability of $99.9 \%$ that the two samples are not extracted from the same parent population. We then divided the area around the cluster into concentric circular annuli such that each annulus had roughly an equal number (nine RGBs in each bin) of reference stars (similar to Lanzoni et al. 2007; Beccari et al. 2013). Figure 5 plots the number of BSSs with respect to that of the reference stars in each annulus, as a function of the distance from the cluster centre expressed in terms of $r_{\mathrm{c}}$ calculated in Sect. 4. It is clearly bimodal, with a peak of BSSs in the inner region, a clear dip at intermediate radii, and an increasing value that is 


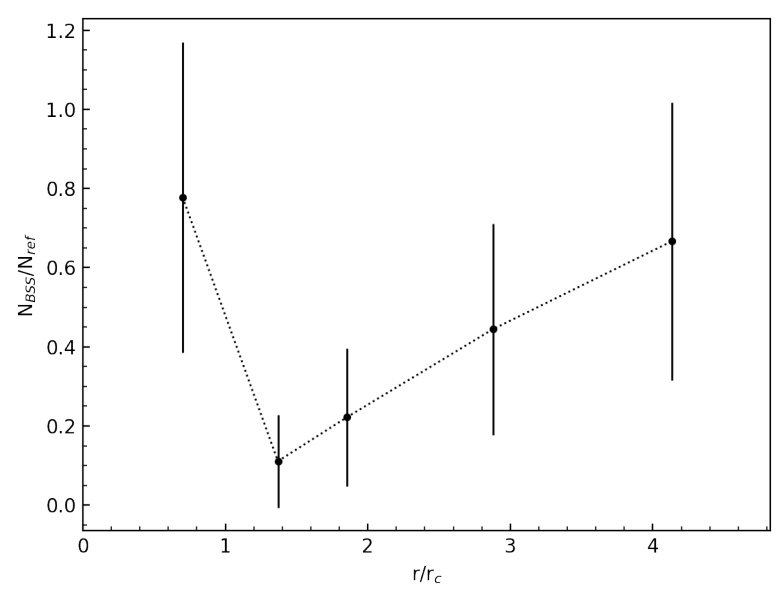

Fig. 5. Number of BSSs with respect to that of the reference stars, plotted as a function of the distance from the cluster centre expressed in terms of $r_{\mathrm{c}}$. The errors are Poisson errors.

slightly smaller than the central one in the outskirts. The minimum occurs at $r_{\text {min }} \sim 3.12^{\prime}$ or $r_{\text {min }} / r_{\mathrm{c}} \sim 1.37$. The BSSs are more concentrated in the centre, with the central radial bin having seven BSSs while the second bin with the minima having just one BSS.

\subsection{Minimum spanning tree}

As an alternative test to evaluate the degree of BSS segregation, in view of the relatively small number of BSSs, we applied the method of the minimum spanning tree (MST; Allison et al. 2009, and references therein). The MST is the unique set of edges (straight lines) connecting a given sample of vertices (here, the star coordinates) without closed loops, to minimize the sum of the edge lengths. The star coordinates are treated as Cartesian points on a plane so the edge lengths are not the same as the distances between the stars on the sky. The length of the MST, $\ell_{\mathrm{MST}}$, is the sum of all such edge lengths connecting the vertices. It is a measure of the compactness of a given sample of vertices and is independent of the centre of the sample. A sample of vertices that is more concentrated on a plane would have a lower $\ell_{\text {MST }}$ than a sample of vertices that is more spread out on a plane. We compared the segregation of the BSSs with respect to a reference population, the RGB stars, by their $\ell_{\text {MST }}$ lengths (e.g. Beccari et al. 2012) within $4^{\prime}$ of the cluster centre. From Fig. 5, one would expect that the BSSs are more concentrated than the reference population within this radius and would thus have a lower $\ell_{\text {MST }}$ than the reference population. We computed $\Gamma_{\text {MST }}$ as the ratio of the MST lengths of the reference population to that of the BSS. We report this dimensionless ratio, which would be greater than 1 if the BSSs are more concentrated than the reference population. We reported the $\ell_{\mathrm{MST}}$ in arbitrary units of length.

We obtained $\ell_{\text {MST }}^{\text {BSS }}=570.8$ for the eight BSSs located within $4{ }^{\prime}$. We then randomly extracted 1000 sets of eight stars from the reference population, and computed the $\ell_{\text {MST }}^{\text {ref }}$ of each set. The $\ell_{\text {MST }}^{\text {ref }}$ distribution is shown in Fig. 6, from which the mean MST length, $\left\langle\ell_{\mathrm{MST}}^{\mathrm{ref}}\right\rangle=736.33$, and standard deviation, $\Delta \ell_{\mathrm{MST}}^{\mathrm{ref}}=97.04$, of the distribution are derived. The level of BSS segregation with respect to the reference stars and its associated uncertainty have been estimated as

$$
\Gamma_{\mathrm{MST}}=\left\langle\ell_{\mathrm{MST}}^{\mathrm{ref}}\right\rangle / \ell_{\mathrm{MST}}^{\mathrm{BSS}}=1.29, \Delta \ell_{\mathrm{MST}}=\Delta \ell_{\mathrm{MST}}^{\mathrm{ref}} / \ell_{\mathrm{MST}}^{\mathrm{BSS}}=0.17 .
$$

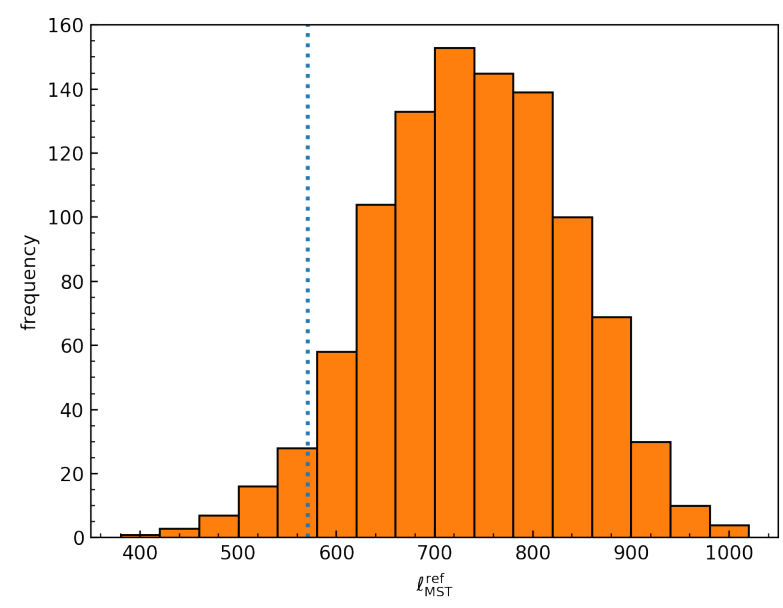

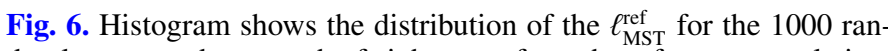
domly extracted sets, each of eight stars, from the reference population. The dotted blue line shows the $\ell_{\mathrm{MST}}^{\mathrm{BSS}}$.

Obtaining a $\Gamma_{\text {MST }}>1$ clearly shows that the BSSs are more concentrated than the reference population within $4^{\prime}$. This bolsters the notion that the radial distribution of the BSSs in Be17 can indeed be used as a "dynamical clock" for the cluster.

\section{Discussion}

From the exquisite data available from GDR2, we are able to identify the members of Be17, including the BSSs. Our stringent selection criteria mean that we may miss main sequence members and not the BSS or giant branch members, both of which should be mostly complete and equally affected by incompleteness. For the brightest cluster members, $G<15 \mathrm{mag}$, we obtain the mean proper motion of the cluster as $\mu_{\alpha} \cos \delta=$ $2.59 \pm 0.26$ mas yr$^{-1}, \mu_{\delta}=-0.26 \pm 0.38$ mas yr$^{-1}$, similar to what we get for the 12 previously identified giants but offset in $\mu_{\delta}$. We observe a spread in the giant branch colour, present also for those stars with GDR2 spectra and hence most certainly members. Such a feature can be caused by a metallicity spread in the giant branch stars, differential reddening in the cluster, or the presence of multiple stellar populations. A significant spread in the metallicity was not observed by Scott et al. (1995) for their 12 giants and the cluster, located below the Galactic plane towards the Galactic anti-centre is not expected to show substantial differential reddening sufficient to cause the spread in colour. Signs of multiple stellar populations have been observed in the open cluster NGC 6791 (Geisler et al. 2012) from $\mathrm{Na}$ abundance measurements. Being an old open cluster, we cannot rule out the presence of multiple stellar populations in Be17 that may be responsible for the colour spread in the giant branch stars. Spectroscopic studies of the giant branch stars of Be17 would be required to check for the presence of multiple stellar populations.

The central concentration of BSSs in the core of Be17 corroborates the massive nature of BSSs compared to the giant branch stars, and hence their suitability as efficient test particles to infer the dynamical state of stellar clusters. It lends support to the identified BSSs being rejuvenated massive main sequence stars, making Be17 a new laboratory to test BSS formation theories with spectroscopic studies. BSS radial distribution has been used as a powerful tool to shed light on the internal dynamical evolution of globular clusters (Ferraro et al. 2012; 
Beccari et al. 2013). In this work we present the first example of its application for an open cluster. Mass segregation in the core of Be17 (Bhattacharya et al. 2017) already indicated that it was dynamically evolved. Using the radial distribution of the BSSs we can, for the first time, make a direct comparison of the dynamical state of an open cluster with that of globular clusters. The bimodal distribution clearly places Be17 with the Family II globular clusters, where the BSSs have just started to sink towards the centre. This is an empirical proof that Be17 is still undergoing dynamical evolution, just like Family II globular clusters, which implies that the distribution of stars in $\mathrm{Be} 17$ is not dominated by its primordial mass distribution. With a lower stellar density than globular clusters, it is easier to obtain radial velocity measurements of the BSS in Be17. This would allow us to find their trajectories thereby utilizing them as kinematic tracers to better understand its dynamical evolution, which would also be characteristic of Family II globular clusters.

It is expected that old open clusters are dynamically evolving and their BSS population, wherever present, can be used to determine their state of dynamical evolution. Ahumada \& Lapasset (2007) indeed observe a significant number of BSS candidates, albeit without reliable membership determination, in many old open clusters whose radial distribution can provide an insight into the dynamical evolution of old open clusters. Since BSSs can only be accurately determined after reliable membership determination, many old open clusters previously classified as being rich in BSSs would need to be re-evaluated with membership determination (Vaidya et al., in prep.). This is important to understand the environmental effects of BSS formation in old open clusters by comparing old open clusters rich in BSSs to those that are deficient in BSSs (e.g. Lee \& Chang 2017).

Acknowledgements. The authors are grateful to the anonymous referee for the valuable comments. SB acknowledges support from the IMPRS on Astrophysics at the LMU Munich. This work has made use of data from the European Space Agency (ESA) mission Gaia (https://www. cosmos.esa.int/gaia), processed by the Gaia Data Processing and Analysis Consortium (DPAC, https : // wwW. cosmos.esa.int/web/gaia/dpac/consortium). This research made use of Astropy, a community-developed core Python package for Astronomy (Astropy Collaboration 2013), Numpy (Oliphant 2015), and Matplotlib (Hunter 2007). This research also made use of NASA's Astrophysics Data System (ADS, https://ui.adsabs.harvard.edu).

\section{References}

Ahumada, J. A., \& Lapasset, E. 2007, A\&A, 463, 789

Allison, R. J., Goodwin, S. P., Parker, R. J., et al. 2009, MNRAS, 395, 1449 Astropy Collaboration (Robitaille, T. P., et al.) 2013, A\&A, 558, A33 Bailer-Jones, C. A. L., Andrae, R., Arcay, B., et al. 2013, A\&A, 559, A74 Bailer-Jones, C. A. L., Rybizki, J., Fouesneau, M., Mantelet, G., \& Andrae, R. 2018, AJ, 156, 58

Beccari, G., Lützgendorf, N., Olczak, C., et al. 2012, ApJ, 754, 108 Beccari, G., Dalessandro, E., Lanzoni, B., et al. 2013, ApJ, 776, 60 Bertelli Motta, C., Pasquali, A., Caffau, E., \& Grebel, E. K. 2018, MNRAS, 480, 4314

Bhattacharya, S., Mishra, I., Vaidya, K., \& Chen, W. P. 2017, ApJ, 847, 138 Bragaglia, A., Tosi, M., Andreuzzi, G., \& Marconi, G. 2006, MNRAS, 368, 1971 Bressan, A., Marigo, P., Girardi, L., et al. 2012, MNRAS, 427, 127 Cantat-Gaudin, T., Jordi, C., Vallenari, A., et al. 2018, A\&A, 618, A93 Carraro, G., Vázquez, R. A., \& Moitinho, A. 2008, A\&A, 482, 777 Chen, W. P., Chen, C. W., \& Shu, C. G. 2004, AJ, 128, 2306 Chen, Y., Girardi, L., Bressan, A., et al. 2014, MNRAS, 444, 2525 Chen, W. P., Bhattacharya, S., Mishra, I., Vaidya, K., \& Lalchand, B. 2017, J. Phys. Conf. Ser., 869, 012093

Dalessandro, E., Beccari, G., Lanzoni, B., et al. 2009, ApJS, 182, 509

Davies, M. B., Piotto, G., \& de Angeli, F. 2004, MNRAS, 349, 129 Dias, W. S., Monteiro, H., Caetano, T. C., et al. 2014, A\&A, 564, A79 Evans, D. W., Riello, M., De Angeli, F., et al. 2018, A\&A, 616, A4 Ferraro, F. R., Beccari, G., Dalessandro, E., et al. 2009, Nature, 462, 1028 Ferraro, F. R., Lanzoni, B., Dalessandro, E., et al. 2012, Nature, 492, 393 Friel, E. D., Janes, K. A., Tavarez, M., et al. 2002, AJ, 124, 2693 Gaia Collaboration (Brown, A. G. A., et al.) 2018, A\&A, 616, A1 Geisler, D., Villanova, S., Carraro, G., et al. 2012, ApJ, 756, L40 Hills, J. G., \& Day, C. A. 1976, ApJ, 17, L87

Hunter, J. D. 2007, Comput. Sci. Eng., 9, 90

Kaluzny, J. 1994, Acta Astron., 44, 247

Katz, D., Sartoretti, P., Cropper, M., et al. 2019, A\&A, 622, A205

Kharchenko, N. V., Piskunov, A. E., Schilbach, E., Röser, S., \& Scholz, R. D. 2013, A\&A, 558, A53

King, I. 1962, AJ, 67, 471

Krusberg, Z. A. C., \& Chaboyer, B. 2006, AJ, 131, 1565

Lanzoni, B., Dalessandro, E., Ferraro, F. R., et al. 2007, ApJ, 663, 267

Lee, H.-U., \& Chang, H.-Y. 2017, J. Korean Astron. Soc., 50, 51 Lindegren, L., Hernández, J., Bombrun, A., et al. 2018, A\&A, 616, A2

Marigo, P., Girardi, L., Bressan, A., et al. 2017, ApJ, 835, 77

Mathieu, R. D., \& Geller, A. M. 2009, Nature, 462, 1032

McCrea, W. H. 1964, MNRAS, 128, 147

Oliphant, T. E. 2015, Guide to NumPy, 2nd edn. (USA: CreateSpace Independent Publishing Platform)

Phelps, R. L. 1997, ApJ, 483, 826

Salaris, M., Weiss, A., \& Percival, S. M. 2004, A\&A, 414, 163

Sandage, A. R. 1953, AJ, 58, 61

Scott, J. E., Friel, E. D., \& Janes, K. A. 1995, AJ, 109, 1706

Sills, A., Glebbeek, E., Chatterjee, S., \& Rasio, F. A. 2013, ApJ, 777, 105

Yen, S. X., Reffert, S., Schilbach, E., et al. 2018, A\&A, 615, A12 